\title{
Influence of internal medicine perioperative consult team on vascular surgical patients
}

\author{
Abhiram Cherukupalli ${ }^{1}$, Simran Parmar ${ }^{1}$, Yuda Shih ${ }^{1}$, Abdalla Butt ${ }^{2}$, Gary K.Yang ${ }^{2}$, Jonathan D Misskey and York N Hsiang ${ }^{2 *}$ \\ ${ }^{1}$ Faculty of Medicine, Department of Surgery, University of British Columbia, Vancouver, BC \\ ${ }^{2}$ Division of Vascular Surgery, Department of Surgery, University of British Columbia, Vancouver, BC
}

\begin{abstract}
Background: In 2015, an Internal Medicine Perioperative Consult Team (IMPCT) was created in our hospital. We sought to determine whether they improve perioperative care without unnecessarily delaying surgery.

Objectives: To evaluate the IMPCT service on vascular surgery patient outcomes and course in hospital.

Methods: Using a prospectively maintained database, we compared vascular surgery patients who received a preoperative IMPCT consult with age, sex, co-morbidities, and surgical intervention matched patients who received no IMPCT consult. The outcomes of interest were post-operative complications, delays in surgery, and length of stay.

Results: From 2015-2017, 71 IMPCT and 129 control patients were identified. The average age ( 73.7 vs. 74.5 years, $p=0.57)$ and male gender $(66 \%$ vs.70\%, $\mathrm{p}=0.60)$ between the two groups were similar. Deaths in the IMPCT and control groups were not different, 4 vs. $5, \mathrm{p}=0.57$. Post-operative complications occurred in $59 \%$ of IMPCT patients compared with $19 \%$ of control patients $(p<0.01)$ due to more cardiac, renal and delirium issues, $p<0.01$. Delays in surgery occurred in $62 \%$ of IMPCT and $40 \%$ control patients, $\mathrm{p}<0.01$. The need for medical optimization in IMPCT (34\%) versus control (6\%) patients was the primary reason for delay, $\mathrm{p}<0.01$. However, the average days of delay to OR was not different ( 4.9 vs. 4.9, $\mathrm{p}=0.97)$ between the two groups, nor was the average length of stay for the two groups, (16.2 vs. 9.5 days, $\mathrm{p}=0.16$ ).
\end{abstract}

Conclusion: More postop complications and delays to surgery were seen in patients who had an IMPCT consult compared with matched controls. In-hospital death, average days of delay to surgery and length of stay was not different.

\section{Background}

Surgical interventions carry risks due to the prevalence of comorbidities, urgency, magnitude, type, and duration of the procedure.1,2 For older patients, having a thorough medical workup prior to noncardiac surgery by a multidisciplinary team with internal medicine or hospitalists specialists working with surgeons may benefit patient outcomes but could also delay surgery and remains controversial.3 A study on patients undergoing hip and knee procedures showed that a co-management team of hospitalists and orthopedic surgeons had fewer complications compared with orthopedic surgeons with medical consultation 4

Vascular surgery patients though are typically older, have more severe co-morbidities, and higher postoperative risk. Two trials to address early medical identification and treatment for high risk patients prior to vascular surgery failed to show benefit in decreasing early or late mortality.5,6 Nonetheless, a recent study suggested that a comanagement model of hospitalists and vascular surgeons decreased patient complications including death.7

Recently, across Canada, Internal Medicine Perioperative Consult Teams (IMPCT) have appeared. Their purpose was to optimize medical conditions and initiate interventions to decrease perioperative risk. In 2015, a new IMPCT service was created in our hospital. Prior to this our standard practice of perioperative care was by surgeons with the resident staff and medicine specialist consultation when necessary. As this was a change to our standard practice, we sought to assess the impact of this new service and hypothesised that the IMPCT service would change the rate of adverse post-op complications following major vascular surgery. The specific objectives were to determine the impact of IMPCT on post-op complications, delays to surgery and length of stay.

\section{Methods}

\section{Data collection}

A three year retrospective review of a prospectively maintained database of vascular surgery patients admitted to the Vancouver General Hospital (VGH) from January 1, 2015 to December 31, 2017 was undertaken. VGH is Canada's second largest academic teaching hospital with approximately $1000+$ bed capacity.

Data was extracted from medical records into an encrypted Excel spreadsheet. Demographics, past medical history, recommendations from the IMPCT consult, type of surgery performed, lab and imaging tests ordered, postop outcomes including delays in surgery, reason for delays, postoperative complications, and length of stay were collected. Postoperative cardiac complications included myocardial infarction,

${ }^{\star}$ Correspondence to: Hsiang YN, Department of Surgery, Gordon and Leslie Diamond Health Care Centre, 11263-2775 Laurel Street, Vancouver, B.C. V5Z 1M9, Tel: +1-604-876-5882; E-mail: York.Hsiang@vch.ca

Received: June 18, 2021; Accepted: June 24, 2021; Published: June 28, 2021 
myocardial injury after noncardiac surgery (or MINS), and new onset congestive heart failure $(\mathrm{CHF})$ or atrial fibrillation. Ethics approval was provided by the University of British Columbia Research Ethics Board (REB H17-00840).

We assembled two cohorts for comparative purposes. For the IMPCT cohort, patients had to be admitted during 2015-2017 and received an IMPCT consult preoperatively. An IMPCT consult could be requested by any of the following: an anesthetist in the pre-admission clinic, the attending surgeon or resident. All inpatients who received a preoperative IMPCT consult during this period were included. A second group of matched patients from the same period based on age, sex, co-morbidities, and surgical intervention were identified from the hospital database and served as control. The primary outcomes of interest were postoperative complications, delays to surgery, and length of stay

\section{Statistical analysis}

Categorical and continuous data were collected and compared between the two groups using the chi-square test for categorical data and unpaired t-tests for continuous data. SPSS software (SPSS Statistics 27, IBM) was used for all calculations. Significance was assumed if $\mathrm{p}<0.05$ (two tailed).

\section{Results}

\section{Pre-operative characteristics}

For the period 2015-2017, all 74 patients who had an in-hospital preoperative IMPCT consult were identified. One patient was excluded from the analysis because they did not have surgery while in hospital. An additional two patients were excluded because they did not receive IMPCT consults preoperatively. Based on the 71 IMPCT patients who underwent operations, a matched control group of 129 patients from the same period was identified and compared. The average age (73.7 vs. 74.5 years, $p=0.57)$ and male gender $(66 \%$ vs. $70 \%, p=0.60)$ between the two groups were not different. Co-morbidities including current or past tobacco use, coronary artery disease, congestive heart failure, atrial fibrillation, hypertension, diabetes, dyslipidemia, end stage renal disease or dialysis, and dementia were the same for both groups (Table 1).

\section{Types of operations}

Overall, 200 primary operations were performed, 71in the IMPCT group and 129 in the control group. There were a number of concomitant procedures, e.g. angioplasty during hybrid procedures and minor amputations, in each group. Femdistal bypasses and angioplasty

Table 1. Preoperative characteristics
\begin{tabular}{|l|c|c|c|}
\hline VARIABLE & IMPCT (\%) & CONTROL (\%) & P value \\
\hline Male Sex & 66 & 70 & 0.29 \\
\hline Age, years, mean & 73.7 & 74.5 & 0.57 \\
\hline $\begin{array}{l}\text { Coronary Artery } \\
\text { Disease }\end{array}$ & $36(50.7)$ & $62(48.7)$ & 0.09 \\
\hline $\begin{array}{l}\text { Congestive Heart } \\
\text { Failure }\end{array}$ & $11(15.5)$ & $8(6.2)$ & 0.09 \\
\hline Atrial Fibrillation & $21(29.6)$ & $25(19.3)$ & 0.09 \\
\hline ESRD or Dialysis & $10(14.1)$ & $14(10.9)$ & 0.09 \\
\hline Hypertension & $57(80.3)$ & $106(82.2)$ & 0.09 \\
\hline Diabetes & $36(14.8)$ & $42(9.1)$ & 0.09 \\
\hline Dyslipidemia & $37(50.7)$ & $82(32.6)$ & 0.09 \\
\hline Dementia & $4(5.6)$ & $8(6.2)$ & 0.09 \\
\hline $\begin{array}{l}\text { Current or past } \\
\text { tobacco user }\end{array}$ & $32(45.1)$ & $99(57.5)$ & 0.09 \\
\hline
\end{tabular}

Table 2. Number and type of operations performed in IMPCT and Control groups

\begin{tabular}{|c|c|c|}
\hline Operation & IMPCT (No.) & Control (No.) \\
\hline \multicolumn{3}{|l|}{ Endovascular } \\
\hline EVAR & 11 & 20 \\
\hline Angioplasty & 13 & 20 \\
\hline \multicolumn{3}{|l|}{ Open } \\
\hline AAA & 6 & 15 \\
\hline Femdistal & 14 & 29 \\
\hline CEA & 8 & 20 \\
\hline Iliofem & 1 & 4 \\
\hline Other & 18 & 21 \\
\hline Total & 71 & 129 \\
\hline
\end{tabular}

$\mathrm{EVAR}=$ endovascular aneurysm repair, $\mathrm{AAA}=$ abdominal aortic aneurysm, $\mathrm{CEA}=$ carotid endarterectomy, other = amputation, debridement, and skin graft

Table 3. Complication rates of IMPCT and Control Patients

\begin{tabular}{|l|c|c|c|}
\hline COMPLICATION & IMPCT (\%) & CONTROL (\%) & P value \\
\hline Death & $4(6 \%)$ & $5(4 \%)$ & 0.57 \\
\hline Cardiac & $22(31 \%)$ & $13(10 \%)$ & 0.01 \\
\hline Respiratory & $6(8 \%)$ & $5(4 \%)$ & 0.17 \\
\hline Renal & $26(37 \%)$ & $14(11 \%)$ & 0.01 \\
\hline Delirium & $12(17 \%)$ & $5(4 \%)$ & 0.01 \\
\hline Sepsis & $1(1 \%)$ & $4(3 \%)$ & 0.46 \\
\hline
\end{tabular}

(with or without stenting) were the most common procedures in each group. The type and number of procedures for each group is displayed in Table 2.

\section{Postoperative complications}

A total of 114 complications occurred in both groups combined. For the IMPCT group, 42 patients had complications (42/71, 59\%) compared with 25 patients $(25 / 129,19 \%, \mathrm{p}<0.01)$ in the control group. Nine patients died before discharge, $4(6 \%)$ in the IMPCT group and 5 (4\%) in the control group, $\mathrm{p}=0.57$. The IMPCT group had significantly more cardiac abnormalities including MI, MINS, new onset CHF and atrial fibrillation ( $31 \%$ vs. $10 \%, \mathrm{p}<0.01)$, renal injuries ( $37 \%$ vs. $11 \%$, $\mathrm{p}<0.01)$, and delirium $(17 \%$ vs. $4 \%, \mathrm{p}<0.01)$. Sepsis occurred in $1(1 \%)$ patient from the IMPCT group and $4(3 \%)$ patients from the control group, $\mathrm{p}=0.46$ (Table 3 ).

\section{Delays in surgery}

There were a number of reasons why inpatients who required surgery could be delayed. These included the requirement for additional imaging, patient related issues such as consent, timing of hospital admission (morning or night), status of OR booking (immediate or urgent), and need for additional medical consults with resultant investigations or management, e.g. BP control. The net result is that one or more of these factors may delay the timing of surgery.

Delays in surgery occurred in $62 \%$ of IMPCT and $40 \%$ control patients, $\mathrm{p}<0.01$. The need for medical optimization in IMPCT $(34 \%)$ versus control $(6 \%)$ patients was the primary reason for delay, $\mathrm{p}<0.01$. Other reasons including neurology referral, OR booking, patient related issues, imaging, and overnight admission were not different (Table 4). However, the average days of delay to OR was not different (4.9 vs. 4.9, $\mathrm{p}=0.97$ ) between the two groups

\section{Length of stay}

For the entire group, the average length of stay was 12.8 days. The IMPCT group had a higher length of stay, 16.2 days, although this was not significantly different than the control group, 9.5 days $(\mathrm{p}=0.16)$. 
Table 4. Reasons for Delay to Surgery

\begin{tabular}{|l|c|c|c|}
\hline Reasons for Delay & IMPCT (\%) & CONTROL (\%) & P value \\
\hline Medical optimization & $24(33 \%)$ & $8(6 \%)$ & 0.01 \\
\hline Neurology consult & $4(6 \%)$ & $9(7 \%)$ & 0.71 \\
\hline OR booking & $5(7 \%)$ & $6(5 \%)$ & 0.48 \\
\hline Patient related & $3(4 \%)$ & $5(4 \%)$ & 0.9 \\
\hline Imaging & $3(2 \%)$ & $4(3 \%)$ & 0.68 \\
\hline Overnight admission & $7(5 \%)$ & $20(15 \%)$ & 0.26 \\
\hline
\end{tabular}

\section{Discussion}

For vascular patients, the main cause of surgical mortality and morbidity is cardiac with event rates of 5-15\% [1-9]. The recognition of the high prevalence of coronary artery disease in peripheral artery patients has led to strategies of preoperative revascularization $[10,11]$ and stratification models [12-16] to identify and treat high risk patients. Additional preoperative testing, however, carries the risk of delaying timely surgery and increases cost [17]. Two randomized studies examined the need for preoperative cardiac revascularization prior to vascular surgery and failed to show benefit $[5,6]$. Despite these trials, revascularization for high-risk noncardiac surgery continues preoperatively in $5 \%$ of patients $[18,19]$.

Recognizing that preoperative revascularization may benefit high risk patients who can delay surgery for one year or more, does a comanagement team lead to improved outcomes or does it unnecessarily delay time to surgery and hospital discharge? A meta-analysis of comanagement of surgical patients by either internal medicine physicians or hospitalists, showed that having a co-management team did not alter length of stay but may benefit in-hospital mortality although the evidence for this was poor. Of the 14 suitable studies only one was a randomized clinical trial and further studies were recommended [3]. For vascular surgery, Iberti, et al. showed that the introduction of a co-management model of hospitalists and vascular surgeons over two years led to a mortality decrease from $2.0 \%$ to $1.0 \%, \mathrm{p}=0.049$, but the length of stay and readmission rates were unchanged [7].

Unlike the Iberti, et al. study which had no concurrent control group, this study using matched controls over a three-year period, had opposite findings. Patients who had IMPCT consults had significantly more cardiac, renal, and delirium complications, and increased delay to surgery primarily due to medical optimization compared with control patients. In addition, in-hospital deaths between the two groups were not different unlike the Iberti, et al. study. However, length of stay and days delayed to surgery was similar to Iberti's findings.

These differences may be explained by two observations, the low event rate and potential selection bias. For the former, over a similar time period, from 2015 to 2018, for 1176 elective open arterial procedures, the perioperative mortality and MI rate was $1.3 \%$ and $2.3 \%$, respectively These outcomes were achieved with standard medical care and few IMPCT consults. For the latter, all patients who had an IMPCT consult during this period were included. Although request for an IMPCT consult came from a surgeon, resident or anesthetist, approximately $24 \%$ of all inpatients had IMPCT consults as many surgeons continued to practice their standard care of treating patients with surgical residents and timely referral to medical specialists. Even though controls were carefully matched by age, sex, co-morbidities and type of operation, a selection bias may still exist as this was a non-randomized study. Future randomized controlled trials should be conducted to answer this issue.

\section{Limitations}

There are a number of limitations. First, it was retrospective although the data was from a prospectively entered database. Second, matched controls (age, sex, co-morbidities, and surgical procedure) were used and although there did not appear to be any major differences between the groups, matching is no substitute for a randomized comparison. Third, selection bias as explained above may have occurred as IMPCT was called upon for certain but not all high risk patients. Fourth, the sample size was small. Fifth, these results apply to only to our service and may not apply to other services in other hospitals. Sixth, no post-discharge outcomes or costs were collected and this should be included in future studies.

\section{Conclusion}

Three years following its introduction, patients seen by the IMPCT team had more complications and delayed surgeries compared with standard care. Future prospective studies to verify these findings should also include long term outcomes and cost analyses.

\section{References}

1. Kristensen SD, Knuuti J, Saraste A (2014) 2014 ESC/ESA Guidelines on non-cardiac surgery: cardiovascular assessment and management. European Heart $J$ 35: 23832431. [Crossref]

2. Fleisher LA, Fleischmann KE, Auerback AD (2014) 2014 ACC/AHA Guideline on Perioperative Cardiovascular Evaluation and Management of Patients Undergoing Noncardiac Surgery. Circulation 30: e278-e333. [Crossref]

3. Shaw M, Pelecanos AM, Mudge AM (2020) Evaluation of internal medicine physician or multidisciplinary team co-management of surgical patients and clinical outcomes: a systematic review and meta-analysis. JAMA Network Open 3: e204088. [Crossref]

4. Huddleston JM, Long KH, Naessens JM (2004) Medical and surgical co-management after elective hip and knee arthroplasty. Ann Intern Med 141: 28-38. [Crossref]

5. McFalls EO, Ward HB, Moritz TE (2004) Coronary-artery revascularization before elective major vascular surgery. $N$ Engl J Med 351: 2795-2804. [Crossref]

6. Poldermans D, Schouten O, Vidakovic R (2007) A clinical randomized trial to evaluate the safety of a noninvasive approach in high-risk patients undergoing major vascular surgery: the DECREASE-V Pilot Study. J Am Coll Cardiol 49: 1763-1769. [Crossref]

7. Iberti CT, Briones A, Gabriels E (2016) Hospitalist-vascular surgery comanagement effects on complications and mortality. Hosp Pract (1995) 44(5):233-236. [Crossref]

8. Zarinsefat A, Henke P (2015) Update in preoperative risk assessment if vascular surgery patients. J Vasc Surg 62: 499-509.

9. Hertzer NR, Beven EG, Young JR (1984) Coronary artery disease in periphera vascular patients. A classification of 1000 coronary angiograms and results of surgical management. Ann Surg 199: 223-33. [Crossref]

10. Rihal CS, Eagle KA, Mickel MC (1995) Surgical therapy for coronary artery disease among patients with combined coronary artery and peripheral vascular disease. Circulation 91: 46-53. [Crossref]

11. Garcia S, McFalls EO (2014) Need for elective PCI prior to noncardiac surgery: High risk through eyes of the beholder. J Am Heart Assoc 3: e001068.

12. Lee TH, Marcantonio ER, Mangione CM (1999) Derivation and prospective validation of a simple index for prediction of cardiac risk of major noncardiac surgery. Circulation 100: 1043-1049. [Crossref]

13. Fleisher LA, Eagle KA (2001) Clinical practice. Lowering cardiac risk in noncardiac surgery. $N$ Engl J Med 345: 1677-1682. [Crossref]

14. Ford MK, Beattie WS, Wijeysundera DN (2010) Systematic review: prediction of perioperative cardiac complications and mortality by the revised cardiac risk index. Ann Intern Med 152: 26-35. [Crossref]

15. Gupta PK, Gupta H, Sundaram A, Kaushik M, Fang X, et al. (2011) Development and validation of a risk calculator for prediction of cardiac risk after surgery. Circulation 124: 381-387. [Crossref]

16. Bertges DJ, Goodney PP, Zhao Y, Schanzer A, Nolan BW, et al. (2010) The Vascular Study Group of New England Cardiac Risk Index (VSG-CRI) predicts cardiac complications more accurately than the Revised Cardiac Risk Index in vascular surgery patients. J Vasc Surg 52: 674-683. [Crossref] 
17. Chassot PG, Delabays A, Spahn DR (2002) Preoperative evaluation of patients with, or at risk of, coronary artery disease undergoing non-cardiac surgery. Br J Anaesth 89 : 747-759. [Crossref]

18. Muthappan P, Smith D, Aronow HD, Eagle K, Wohns D, et al. (2014) The Epidemiology and Outcomes of Percutaneous Coronary Intervention before High-Risk Non-Cardiac Surgery in Contemporary Practice: insights from the BMC2 Registry. J Am Heart Assoc 3: e000388. [Crossref]
19. Hawn MT, Graham LA, Richman JS, Itani KM, Henderson WG, et al. (2013) Risk of Major Adverse Cardiac Events Following Noncardiac Surgery in Patients with Coronary Stents. JAMA 310: 1462-1472. [Crossref]

20. Huang BY, Yang GK, Strandberg S, Misskey J, Hsiang YN (2021) Exploring the utility of brain natriuretic peptide measurement in Vascular Surgery. Ann Vasc Surg 2021 (in press).

Copyright: (2021 Cherukupalli A. This is an open-access article distributed under the terms of the Creative Commons Attribution License, which permits unrestricted use, distribution, and reproduction in any medium, provided the original author and source are credited. 